\title{
The spectroscopic orbits and other parameters of the symbiotic binary FN Sgr ${ }^{\star}, \star \star$
}

\author{
E. Brandi ${ }^{1,2}$, J. Mikołajewska $^{3}$, C. Quiroga ${ }^{1,4}$, K. Belczyński ${ }^{5}$, O. E. Ferrer ${ }^{1,4}$, L. G. García ${ }^{1}$, and C. B. Pereira ${ }^{6}$ \\ ${ }^{1}$ Facultad de Ciencias Astronómicas y Geofísicas, Universidad Nacional de La Plata, Argentina \\ e-mail: ebrandi@fcaglp.unlp.edu.ar \\ 2 Comisión de Investigaciones Científicas de la Provincia de Buenos Aires (CIC), Argentina \\ 3 Copernicus Astronomical Center, Warsaw, Poland \\ ${ }^{4}$ Consejo Nacional de Investigaciones Científicas y Técnicas (CONICET), Argentina \\ 5 Department of Physics and Astronomy, Northwestern University, 2131 Sheridan Road, Evanston, IL 60208 - 2900, USA \\ ${ }^{6}$ Observatório Nacional - Rua Gen. José Cristino, 77, Sao Cristovao - CEP 20921-400, Rio de Janeiro, Brazil
}

\section{Received 15 December 2004 / Accepted 28 April 2005}

\begin{abstract}
We present a study of the eclipsing symbiotic binary FN Sgr with a period of 568.3 days determined photometrically and confirmed spectroscopically. The hot component underwent a 2.5 mag eruption covered by most of our spectroscopic observations. In particular, we have determined for the first time spectroscopic orbits based on the radial velocity curves for both components. A set of blue absorption lines resembling an A-F type star is present in all our spectra and they seem to be associated with the hot component. Based on the light curve, we derive the red giant's radius $\left(140 R_{\odot}\right)$ and the orbital inclination $\left(i=80^{\circ}\right)$. We find that FN Sgr is similar to other S-type symbiotic binaries, composed by an M 5-type giant $\left(M_{\mathrm{g}}=1.5 M_{\odot}\right)$ and a hot white dwarf $\left(M_{\mathrm{h}}=0.7 M_{\odot}, R_{\mathrm{h}}=0.2 R_{\odot}\right)$ with a binary separation of $\sim 1.6 \mathrm{AU}$. The red giant is just filling its Roche lobe and a geometrically and optically thick accretion disk is likely to be present around the low-mass accretor. The evolution of $T_{\mathrm{h}}$ and $L_{\mathrm{h}}$ along the active phase argues in favour of accretion disk instabilities similar to those of $\mathrm{Z}$ And. We have also studied spectral changes and photometric variations as a function of both the hot component activity and the orbital motion.
\end{abstract}

Key words. stars: binaries: eclipsing - stars: binaries: symbiotic - stars: fundamental parameters - stars: individual: FN Sgr

\section{Introduction}

Symbiotic stars are interacting binaries in which an evolved giant star transfers material to a much hotter compact companion. The typical configuration of a symbiotic binary is a red giant transferring material, via stellar wind, to a white dwarf. In some cases a red giant is replaced by a yellow giant or a carbon star, and a white dwarf by a main sequence or a neutron star. Most symbiotic stars $(\sim 80 \%)$ contain a normal giant star, and based on their near-IR colors (showing presence of stellar photospheres of $T_{\mathrm{eff}} \sim 3000-4000 \mathrm{~K}$ ) they are classified S-type systems (stellar). The remaining symbiotics contain a Mira variable, and their near-IR colors give temperatures of $\sim 1000 \mathrm{~K}$ indicating the presence of warm dust shells, and they are classified as D-type (dusty). These two types seem to

* Based on observations taken at Complejo Astronómico El Leoncito (CASLEO), operated under an agreement between the Consejo Nacional de Investigaciones Científicas y Técnicas de la República Argentina, the Secretaría de Ciencia y Tecnología de la Nación and the National Universities of La Plata, Córdoba and San Juan.

$\star \star$ Tables 1-3 are only available in electronic form at http://www. edpsciences.org originate from different orbital separations of components. For large separations (long periods) the cool star was able to evolve to the Mira state producing a dust shell around the star. On the other hand for smaller separations (shorter periods) we find normal giants.

Although we know of about 188 symbiotic stars, and another 28 are suspected to be symbiotic (Belczyński et al. 2000), only around 40 systems have known orbital periods and in only 27 systems were spectroscopic orbits determined (Mikołajewska 2003). The majority of the symbiotic systems with known orbital period are S-type, and their periods are generally of the order 200-1000 days. Periods of D-type systems are harder to estimate because they are much longer and are superimposed with pulsations of the Mira variable; for the Mira Symbiotics V1016 Cyg and R Aqr orbital periods of about 80 years (Schild \& Schmid 1996) and 44 years (Hollis et al. 1997) respectively, were estimated. For a detailed review of orbital parameters of symbiotic binaries we refer the reader to Mikołajewska (2003).

This work is part of a major project in which we determine periods and spectroscopic orbits of symbiotic binaries on the basis of spectroscopic data collected over more than ten years. 
In selecting the southern S-type symbiotic star FN Sgr, we chose to use visual photometry from the Variable Star Section Circulars of The Royal Astronomical Society of New Zealand (RASNZ) over 30 years (1972-2002) and high resolution spectra obtained with the $2.15 \mathrm{~m}$ telescope of CASLEO (San Juan, Argentina) during the period 1990-2002.

\section{Observations}

\subsection{Spectroscopy}

Spectroscopic observations were performed with the $2.15 \mathrm{~m}$ "Jorge Sahade" telescope of CASLEO (San Juan, Argentina). During 1990 medium-low resolution spectra were taken with a Boller \& Chivens Cassegrain spectrograph using a photoncounting Reticon, called the Z-Machine $(\lambda / \Delta \lambda=4100$ and 2700 in the blue and red filters respectively). Since 1995, high resolution spectra were obtained with a REOSC echelle spectrograph using a Tek CCD $1024 \times 1024$ pixels $(\lambda / \Delta \lambda=$ $15000)$.

Three low resolution spectra were taken with the $1.52 \mathrm{~m}$ telescope of ESO at La Silla (Chile) and the B and C spectrograph used in the spectral ranges $\lambda \lambda 3450-7425$ and 3099$5098 \AA$ A. Table 1 shows a log of the observations. Barbá et al. (1992) detail the Z-Machine acquiring and reducing data procedures. The CCD data were reduced with $\operatorname{IRAF}^{1}$ packages, CCDRED and ECHELLE and all the spectra were measured using the SPLOT task within IRAF.

To obtain the flux calibration, standard stars from Hamuy et al. (1992) and Hamuy et al. (1994) were observed each night. A comparison of the spectra of the standards suggests that the flux calibration errors are about 15 per cent for the Z-Machine and 20 per cent in the central part of each order for the REOSC echelle images, respectively.

In our analysis we have included the flux and radial velocity measurements of the $\mathrm{H} \alpha$, He II $\lambda 4686$ and [O III] $\lambda 5007$ emission lines given by Van Winckel (1993), corresponding to July $1988(\sim \phi=0.88)$ and September $1989(\phi=0.62)$.

\subsection{Photometry}

The main source of photometric data in this work consists of mean visual estimates published in the Variable Star Section Circulars of The Royal Astronomical Society of New Zealand in the years 1972-2002. We have also estimated $V$ magnitudes by integration and convolution of the spectra with the standard $V$ filter response function. These estimations, $V_{\mathrm{sp}}$, are included in the last row of Table 2 and its accuracy is of the order of $\pm 0.2 \mathrm{mag}$. Collected visual and $V$ photometry is shown in Fig. 1. The light curve of FN Sgr (JD 2441500 2452600 ) shows a systematic brightness decline with moderate periodic-like light changes, and with one major eruption around JD 2451000.

${ }^{1}$ IRAF is distributed by the National Optical Astronomy Observatories, which is operated by the association of Universities for Research in Astronomy, INC., under contract to the National Science Foundation.

\section{Analysis and discussion}

\subsection{Orbital period}

We have analyzed the RASNZ visual photometry using the period-search method described by Schwarzenberg-Czerny (1997). The periodogram shown in Fig. 2 has been obtained from all visual data presented in Fig. 1. We obtained two significant frequency peaks $(\sim 3 \sigma)$ which occur at $\sim 0.0018 \mathrm{day}^{-1}$ and $\sim 0.0003$ day $^{-1}$, respectively. The highest frequency peak represents light changes with an amplitude of about 0.7 mag and a period of 568 days while the lower peak corresponds to a period $\sim 3100$ days which is more than half of the time interval covered by our observations. It is also induced by the $2.5 \mathrm{mag}$ outburst around JD 2451000 seen in Fig. 1. To construct the phase diagram, we divided observational points into two groups: those from the quiescent phase (JD $2441500-2449977$ ) and those from the active phase (JD 2450007 - 2452 000). Points from the quiescent phase were corrected for the brightness decline to make the periodic light changes more visible. We phased both groups using

$\operatorname{Min}=\mathrm{JD} 2450270( \pm 2)+568.3( \pm 0.3) \times E$.

and then we constructed normal points which are shown in Fig. 2. On both phased light curves we see a similar trend: a clearly pronounced primary minimum $(\varphi \sim 0.0)$, and a small dip around $\varphi \sim 0.5$. The amplitude of the active light curve $(\sim 2.5 \mathrm{mag})$ is more than three times larger than that of the quiescent light curve ( $\sim 0.7 \mathrm{mag})$. A similar behaviour is observed in the eclipsing symbiotic systems of CI Cyg (Mikołajewska 2001, and references therein) and AX Per (Mikołajewska \& Kenyon 1992), and it is consistent with eclipses of the active hot component by the giant companion. The photographic light curve of FN Sgr from 1918 to 1927 (Payne 1928) shows a clear light minimum around JD 2424700 , which corresponds, according to Eq. (1), to phase $\phi=0.01$, and thus seems to confirm our ephemeris. This suggests that whatever causes these periodic light changes was also present 70 years ago and it is a long term phenomenon. The radial velocity, emission line flux and $[\mathrm{TiO}]_{1}$ index variations all show the same periodicity, which we attribute to the orbital period. In all cases, the most regular changes have been obtained with the 568.3-day period. As is observed in all well-studied symbiotic stars, the orbitally related photometric changes are accompanied by some spectral variability such as changes in the optical emission line fluxes and in the relative depths of $\mathrm{TiO}$ absorption molecular bands.

\subsection{Spectral variability}

In Table 2 we list the emission line fluxes, obtained by integrating the line profile above the linearly interpolated continuum. We have also measured the relative depth of the $\mathrm{TiO} \lambda 6180$ absorption band, to obtain the $[\mathrm{TiO}]_{1}$ molecular index and the spectral type of the cool component of FN Sgr, according to the calibration given by Kenyon \& Fernandez-Castro (1987).

Most of our spectroscopic observations were taken during the optical outburst phase (Fig. 1). The outburst behaviour is similar to that observed in other classical symbiotic stars (e.g. 


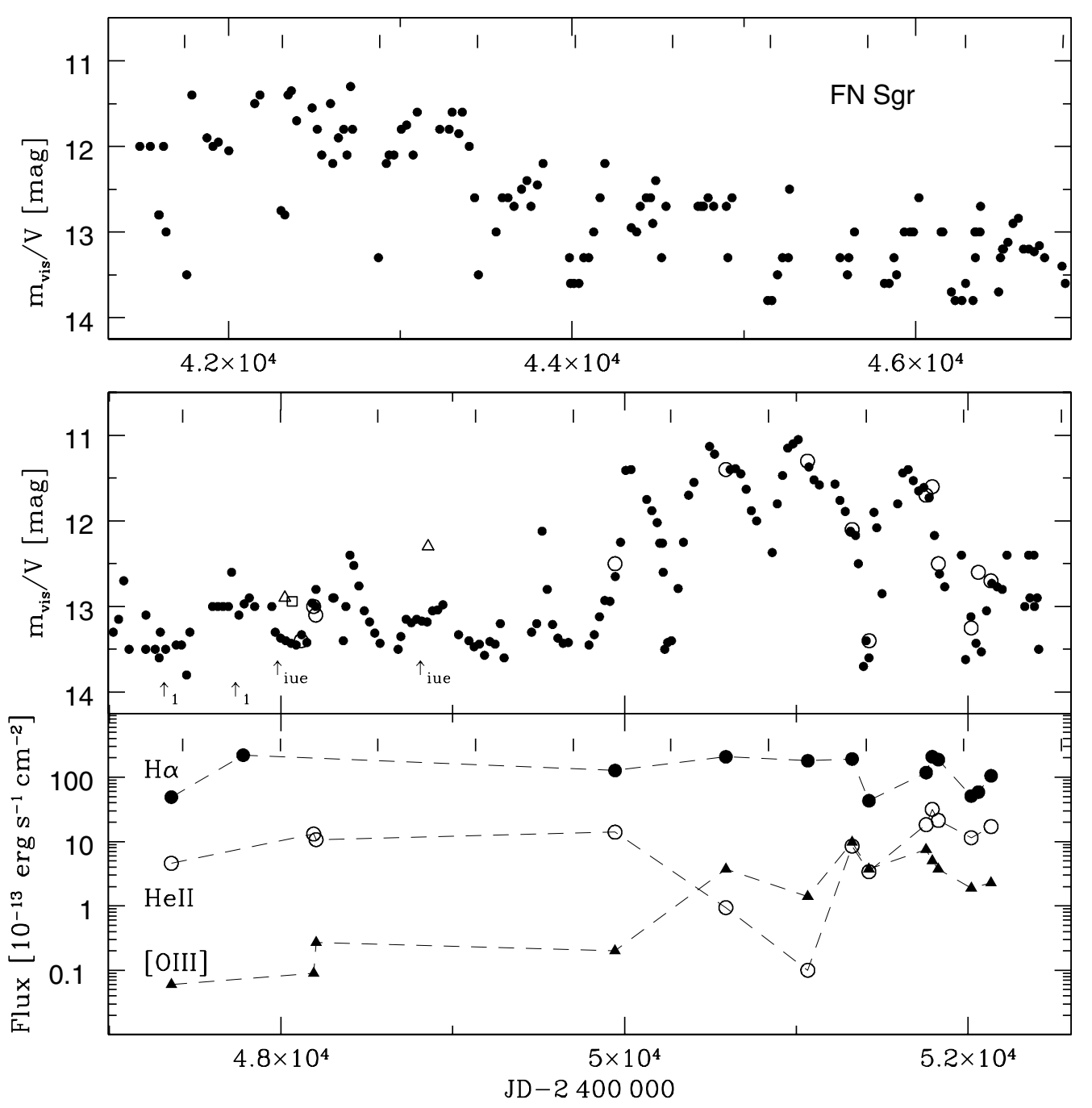

Fig. 1. (Top) visual light curve of FN Sgr in 1972-2002. Dots: visual observations from RASNZ, open circles: $V$ magnitudes calculated from our spectra and those from Barbá et al. (1992), square: from Munari et al. (1992), and triangles: derived from FES counts. (Bottom) evolution of emission line fluxes of $\mathrm{H} \alpha$, He II 4686 and [O III] 5007 (shifted by 0.5 for clarity) in 1988-2002. Bars mark times of photometric minima/times of the inferior spectroscopic conjunction of the red giant. Labelled arrows point to Van Winckel et al. (1993) data (1) and the available IUE spectra.

CI Cyg and AX Per). The rise in optical brightness was first accompanied by a large increase in the [O III] emission lines and broadening of the emission line wings (Fig. 3). The permitted HI and He I emission lines were also increasing although not as much as the forbidden lines, whereas He II 4686 was decreasing. Then, near the visual maximum (1996-1998) the [O III] emission decreased, and He II 4686 practically disappeared. At the same time the permitted emission lines have maximum widths. In 1999, the visual brightness gradually declined with increasing the [O III] and He II emission lines.

The [O III] nebular emission reached maximum intensity in May 1999, and after that it declined following the visual magnitudes. He II 4686 emission reached maximum intensity in September-October 2000, somewhat later than the forbidden line emissions. On the other hand, the forbidden line $[\mathrm{Fe}$ VII] 26087 was absent during the outburst, and then the intensity increased since July 2000, reaching maximum in April 2002.
In Fig. 4 we present a few emission line fluxes and the $[\mathrm{TiO}]_{1}$ index variations as a function of the orbital phase. The $[\mathrm{TiO}]_{1}$ indices are much deeper near the photometric minimum than around the maxima, indicating a spectral type as late as M5 III. All this suggests that the continuum level changes due to occultation of both the hot component and part of the nebulosity by the $\mathrm{M}$ giant companion during the minima and that the spectral type of the giant is earlier during the maxima indicating strong contamination by the hot component and the ionized nebula.

The H I, He I and He II fluxes decreased in intensity around primary minima. The emission line He II $\lambda 4686$ decreased in intensity by roughly a factor of two and it suffers narrower eclipses than do HI and He I lines (Fig. 4). Although the forbidden line fluxes [O III] and [Fe VII] show significant variations along the optical brightness they do not change during the photometric minima suggesting that these emission lines are formed in an extended region. This behavior 

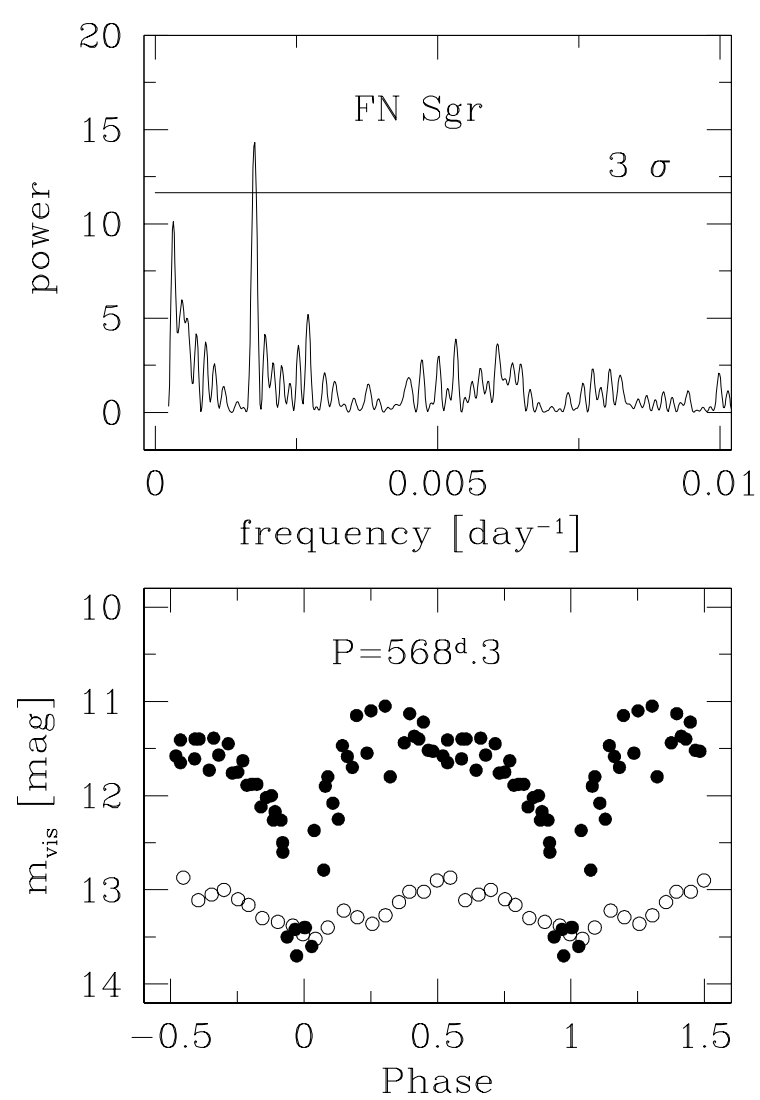

Fig. 2. Periodogram (top) and phased light curves (bottom) for FN Sgr. Open circles represent normal points calculated from quiescent light curve (MJD 45 000-49 500, dots represent the light curve from the recent outburst (MJD 50 000-52 000).

parallels that observed in CI Cyg (Kenyon et al. 1991) and AX Per (Mikołajewska \& Kenyon 1992).

In general, in the observed members of the Balmer series, the broad emission is cut by a central absorption and the red peak is stronger than the blue one. The $\mathrm{H} \alpha$ emission profiles are shown in the left panel of Fig. 3. In August 1995, April and August 2002 (not shown in Fig. 3) the absorption is very weak or absent and the double peak structure is replaced by an asymmetric single profile. The largest value of the absorption intensity (see Table 2) is observed in September 1998-May 1999, during the maximum of the light curve and no intensity variation with the phases was detected. A similar behavior is presented by the central absortions of $\mathrm{H} \beta$ and $\mathrm{H} \gamma$. The radial velocity of this feature is practicaly constant, $-88 \pm 2 \mathrm{~km} \mathrm{~s}^{-1} ;-78 \pm 1 \mathrm{~km} \mathrm{~s}^{-1}$ and $-77 \pm 0.4 \mathrm{~km} \mathrm{~s}^{-1}$ in $\mathrm{H} \alpha, \mathrm{H} \beta$ and $\mathrm{H} \gamma$ respectively.

\subsection{Spectroscopic orbits}

We present the radial velocity curves for both components of the system FN Sgr. For the cool component we have measured M-type absorption lines at wavelengths longer than $6000 \AA$ corresponding to Fe I, Ti I, Ni I, Si I, O I, Zr I, Co I, V I and Mg I. In the blue region, between $\lambda \lambda 4000-5000 \AA$ we have measured cF-type absorption lines corresponding mainly to Cr II, Fe II, Ti II and Y II. The radial velocity curve of these ions seems to be in anti-phase with that of the cool star. The emission lines of He I, He II, Fe II and forbidden transitions of [O III] and [Fe VII] were also measured. The individual radial velocities were obtained by a Gaussian fit of the line profiles. A mean value was calculated for each spectrum and the resulting heliocentric velocities together with their standard errors and the number of measured lines are given in Table 3.

In addition we have determined the radial velocities from the broad emission line wings of $\mathrm{H} \alpha, \mathrm{H} \beta$ and He II $\lambda 4686$, which would reflect the motion of the hot component if they were formed in the inner region of the accretion disk or near to the hot star. In this way we have applied the same method that Schneider \& Young (1980) and Shafter (1983) succesfuly used in studies of cataclysmic binaries (for more details about the application of this method to symbiotic binaries, see Quiroga et al. 2002). However, the Balmer line wings are at times affected by a mixture of Raman and Rayleigh scattering (Jung \& Lee 2004) and it is therefore not clear if they are associated with the hot component motion. This may explain the large scatter in the radial velocity curves in Fig. 6c.

The $\mathrm{M}$-abs radial velocity variations are consistent with the photometric period over more than seven photometric cycles covered by our data (see Fig. 5), so we adopt $P_{\text {orb }}=568.3$ days. In order to find the other orbital parameters: $T_{0}$, the time of periastron passage (or spectroscopic conjunction for $e=0$ solutions); $\gamma$, the baricentral velocity; $K$, the semi-amplitude and $\omega$, the longitude of periastron, we applied Bertiau's program (1967) based on the Lehmenn-Filhés method, considering both cases, $e=0$ and $e \neq 0$. Table 4 gives the constrained solutions to circular orbits and the best fitting for an eccentric orbit for the indicated groups of absorption and emission lines. $\Delta T=T_{\text {spconj }}-T_{\text {photmin }}$ is the time difference between spectroscopic conjunction and photometric minimum. The circular orbit for the M-abs line fits very well the radial velocity data of the giant (Fig. 6, panel a), but an orbit with a low eccentricity $(e=0.06 \pm 0.03)$ represents the best solution. The times of spectroscopic conjunction coincides with optical eclipses for circular and quasi-circular orbits, so the absorption lines trace the cool component's motion.

The best solution for the cF-type absorption features (as well as that for the He II broad emission line wings) leads to significant eccentricity $(e=0.33)$, whereas the red giant orbit is circular or nearly circular. A similar result was obtained for AR Pav, in which the eccentricity apparently increases with the hot component activity (Quiroga et al. 2002). Moreover, in both FN Sgr and AR Pav the periastron longitude of the elliptical orbit solution for the hot component is near $270^{\circ}$ indicating that the apparent eccentricity may be an effect tied to the line of sight. The cF-type absorption velocities are $180^{\circ}$ out of phase with the M-giant and the systemic velocity is blueshifted by $\sim 5 \mathrm{~km} \mathrm{~s}^{-1}$. The dotted curve in the panel $\mathrm{b}$ of Fig. 6 is the best-fitting circular solution for these data. Combining the semi-amplitudes of the $\mathrm{M}$ giant and the blue absorption component for the circular orbit (Table 4) gives a mass ratio $q=M_{\mathrm{g}} / M_{\mathrm{h}}=2.3 \pm 0.05$, component masses of $M_{\mathrm{g}} \sin ^{3} i=1.3 M_{\odot}$ and $M_{\mathrm{h}} \sin ^{3} i=0.63 M_{\odot}$, and the binary separation $A \sin i=350 R_{\odot}$. On the other hand, considering the combined circular solutions, the $\mathrm{M}$ giant, the blue 


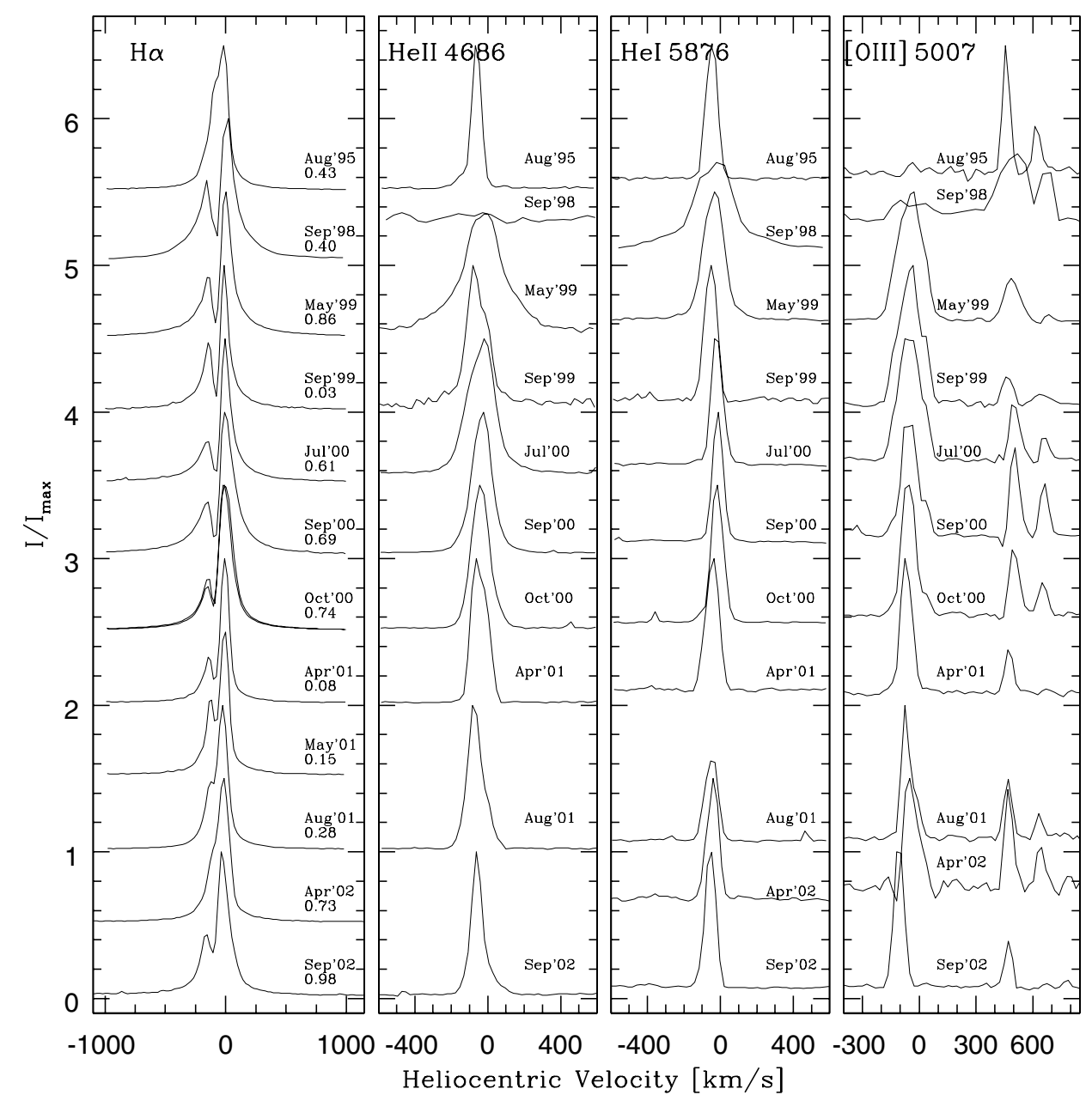

Fig. 3. Emission line profiles in FN Sgr. Each profile was normalized to maximum intensity. The profiles are shifted vertically for better display.

absorptions and the He II wings, $q=M_{\mathrm{g}} / M_{\mathrm{h}}=1.9 \pm 0.08$, $M_{\mathrm{g}} \sin ^{3} i=0.88 M_{\odot}$ and $M_{\mathrm{h}} \sin ^{3} i=0.46 M_{\odot}$ with a binary separation $A \sin i=320 R_{\odot}$. Both the mass ratio and component masses obtained for FN Sgr are similar to those derived for other S-type symbiotic systems (e.g. Mikołajewska 2003, and references therein).

The He I, He II and Fe II emission lines seem also to follow the hot component's motion, but their semi-amplitudes, $\sim 16 \mathrm{~km} \mathrm{~s}^{-1}$ for He I and He II and $9 \mathrm{~km} \mathrm{~s}^{-1}$ for Fe II, are lower than that obtained from the $\mathrm{cF}$-type absorptions. The Fe II curve shows a more negative baricentral velocity $\sim 6 \mathrm{~km} \mathrm{~s}^{-1}$ and a larger difference between the time of the spectroscopic conjunction and the eclipse. In the case of He I and He II, the $\gamma$ velocity $\sim-54 \mathrm{~km} \mathrm{~s}^{-1}$ is nearer to that of the giant and the derived time of spectroscopic conjunction differs from the photometric minimum by around 32 days. Such behavior can be explained, as in AR Pav (Schild et al. 2001; Quiroga et al. 2002), by a contribution from material between the two stars and in the neighborgood of the red giant which can both reduce the amplitude and shift the mean velocity, as for Fe II.

The forbidden lines [O III $] \lambda 5007$ and [Fe VII] $\lambda 6085$ also show variable velocities (see Table 3 ), but their changes do not follow the orbital motion of either binary component. As shown in Fig. 6 panels $g$ and $h$, the radial velocities of $\lambda 5007$ present a deep minimum near the eclipse and the same is observed in the [Fe VII] $\lambda 6085$, but at phases $0.2-0.4$. The profiles of both lines show a double structure at some specific epochs. As can be seen in Fig. 3, the [O III] $\lambda 5007$ profiles present, in general, asymmetries or a weak component to the red side, except at phases 0.08 (April 2001) and 0.98 (September 2002), when the deep minimum in radial velocity is observed. The [Fe VII] $\lambda 6085$ profiles also show two peaks, sometimes very conspicuous with a separation of $\sim 90 \mathrm{~km} \mathrm{~s}^{-1}$, but the profile is single and symmetric at the same phases (0.08 and 0.98).

In order to approximate the individual masses of the FN Sgr system the orbital inclination can be estimated by:

$\cos i \leq \sqrt{\left(R_{\mathrm{t}} / A\right)^{2}-\left(R_{\mathrm{g}} / A\right)^{2}}$

where $R_{\mathrm{g}}$ is the red giant radius and $R_{\mathrm{t}}$ is the mean tidal radius, defined as the radius of a sphere with a volume equal to the Roche lobe volume. $R_{\mathrm{t}} / A=0.45 \pm 0.002$ and $0.43 \pm 0.004$ are obtained from Eggleton (1983) for $q=2.3 \pm 0.05$ and $q=$ $1.9 \pm 0.08$, respectively.

Useful constraints on both the red giant radius $R_{\mathrm{g}}$ and the eclipsed gas $R_{\text {ecl }}$ can be derived from the light curves shown in Fig. 1. 


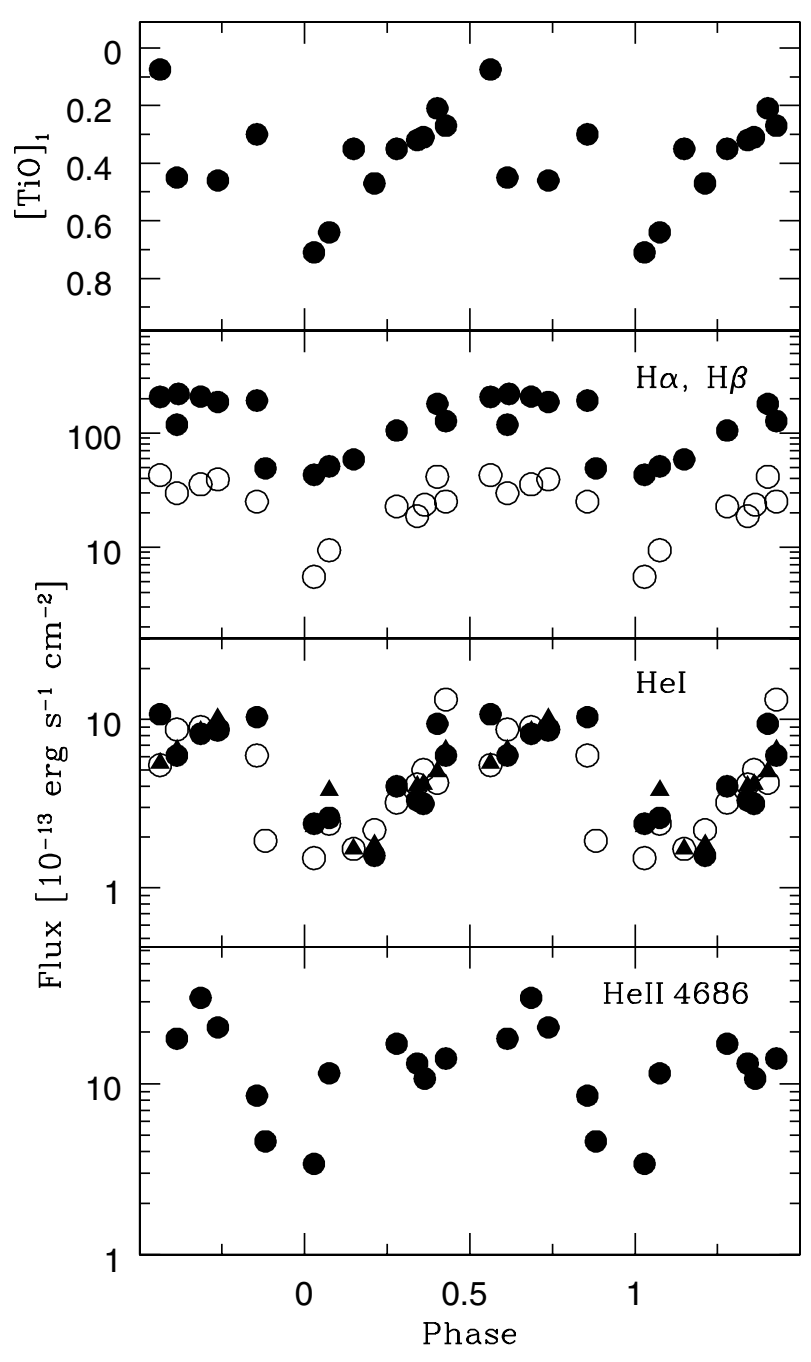

Fig. 4. Phase plot of $[\mathrm{TiO}]_{1}$ indices (top), $\mathrm{H} \alpha$ (dots) and $\mathrm{H} \beta$ (open circles), He I 5876 (dots), 6678 (open circles) and 7065 (triangles) (middle), and He II 4686 emission line fluxes (bottom). The He II 4686 fluxes obtained during the optical outburst maximum, in May 1997 and September 1998, respectively, have been omitted because the very dramatic change in He II flux is due to the outburst and not to orbital motion. The ordinate is logarithmic for line fluxes and linear for [TiO] indices.

If the eclipsed gas volume is centered on the hot star, we can define $\phi_{1}$ and $\phi_{2}$ as the duration of the eclipse and the duration of totality, respectively, and they are related to $R_{\mathrm{g}}$ and $R_{\mathrm{ecl}}$ by

$R_{\mathrm{g}} / A+R_{\mathrm{ecl}} / A=\sin \left(\phi_{1} / 2\right)$

$R_{\mathrm{g}} / A-R_{\mathrm{ecl}} / A=\sin \left(\phi_{2} / 2\right)$

We considered the two deep and well difined eclipses during the outburst at JDM 50251 and JDM 51407. The data suggest $\phi_{1}=80^{\circ} \pm 3^{\circ}$ and $\phi_{2}=23^{\circ} \pm 2^{\circ}$ so $R_{\mathrm{g}} / A=0.42 \pm 0.02$ and $R_{\mathrm{ecl}} / A=0.22 \pm 0.01$. The orbital inclination is then $i=80^{\circ}$, so $M_{\mathrm{g}}=1.5 M_{\odot}$ and $M_{\mathrm{h}}=0.69 M_{\odot}$. The giant's tidal radius, $R_{\mathrm{t}} / A \sim 0.45$, is similar to the apparent red giant radius derived from eclipses, indicating that the red giant in FN Sgr is filling its Roche lobe.

\section{Reddening and distance}

In order to know the physical parameters of FN Sgr we have to obtain a good determination of the interstellar extinction and distance.

The interstellar reddening was derived from $\mathrm{He}$ I, $\mathrm{HI}$ and He II emission line ratios (excluding the spectra taken during the visual maximum when He II lines are very weak or absent), in the same way as Mikołajewska et al. (1997) did.

In particular, $\mathrm{H} \gamma / \mathrm{H} \beta / \mathrm{H} \alpha$ ratios indicate $E_{B-V}=0.3 \pm 0.1$, HeI 6675/5876 and 7065/5876 are consistent with $E_{B-V} \sim$ $0.45 \pm 0.3$ and the He II $4686 / 5411$ ratios indicate $E_{B-V} \leq$ $0.2 \pm 0.1$.

Comparing the He II 4686 mean out-of-eclipse fluxes observed in 1990-95 with the He II 1640 flux measured in the IUE spectrum in 1992, we estimate an upper limit for $E_{B-V} \leq 0.13$ because the He II 1640 line was saturated in the IUE image. These values can be compared with the galactic extinction towards FN Sgr $(l=16.2, b=-9.0)$ which is $0.2 \leq E_{B-V} \leq$ 0.3 (Burstein \& Heiles 1982). For further analysis, we adopt $E_{B-V}=0.2$.

The $[\mathrm{TiO}]_{1}$ index measured during the eclipse indicates an M5 giant, consistent with the $J H K$ colours of FN Sgr (Munari et al. 1992) and $E_{B-V} \sim 0.2$. Using the spectral parallax method for mean dereddened $K_{0}=7.8$, the spectral type M5III, and $M_{K}=-6.4$ (derived from evolutionary tracks for a solar metallicity $1.6 M_{\odot}$ giant) we obtain $d=7 \mathrm{kpc}$. If the cool giant is similar to the galactic buldge giants rather than to the bright local giants, we estimate $d=5 \mathrm{kpc}$. The upper limit to the distance, assuming that the giant fills its tidal lobe, is $d \sim 8 \mathrm{kpc}$ which places FN Sgr in the galactic buldge. Knowing the eclipse radius of the $\mathrm{M}$ giant provides an opportunity to estimate the distance to FN Sgr. Using the Barnes-Evans relation (Cahn 1980),

$F_{K}=4.2211-0.1 K-0.5 \log s$,

with the $K$ surface brightness, $F_{K}=3.83$ (M5III), we estimate the giant's angular diameter in arc milliseconds, $s=0.184$ mas. If the giant's radius is $R_{\mathrm{g}} \sin i=147 R_{\odot}$, a more accurate distance $d \sin i=7.4 \pm 0.4 \mathrm{kpc}$ is obtained. For further analysis, we adopt $d=7.5 \mathrm{kpc}$. Our distance estimate is larger than those given by Munari \& Buson (1994) - $d=3.2 \mathrm{kpc}-$ and Barbá et al. (1992) $-d=2.9 \mathrm{kpc}$. We think that the lower value given by Munari \& Buson (1994) is caused by their assumption of a much higher reddening and earlier spectral type of the giant, whereas Barbá et al. (1992) used a much earlier spectral type (M 1.4).

\section{The nebula}

During the eclipse the giant occults some features of H I, He I and He II at primary minimum (Fig. 4), but does not affect the forbidden lines of [O III] and [Fe VII]. As in CI Cyg and AX Per (Kenyon et al. 1991; Mikołajewska \& Kenyon 1992) we can consider the nebula divided into the eclipsed and the uneclipsed gas.

Almost $90 \%$ of the He II $\lambda 4686$ flux vanishes during the primary minima along the eruption. The duration of this eclipse 


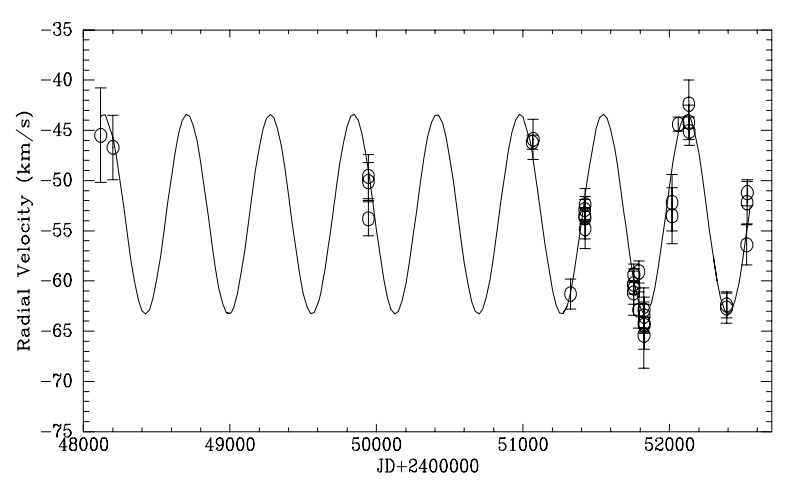

Fig. 5. Circular orbital solution for $P_{\text {orb }}=568.3$ days fitting the heliocentric radial velocity of the red giant in FN Sgr.

$\phi_{1}=152^{\circ} \pm 31^{\circ}$ which combined with $R_{\mathrm{g}} / A=0.42$ gives the fractional radio of the eclipsed region $R_{\mathrm{He}^{2+}} / A=0.55 \pm 0.02$, or $R_{\mathrm{He}^{2+}} \sim 200 R_{\odot}$. With an average integrated line flux during the eclipse, $F(\lambda 4686)=7.3 \times 10^{-13} \mathrm{erg} \mathrm{cm}^{-2} \mathrm{~s}^{-1}$, we estimated the electron density in the He II region as $n_{\mathrm{e}}=10^{10} \mathrm{~cm}^{-3}$ for $T_{\mathrm{e}}=30000 \mathrm{~K}$.

Similar considerations can be made for the eclipsed $\mathrm{H}$ I and He I emission lines which are less deep and broader than those of He II, especially $\mathrm{HI}$, indicating that the region is not completely occulted. We estimated $\phi_{1}=174^{\circ} / 196^{\circ}$ for $\mathrm{H} \mathrm{I}$ and $\mathrm{He}$ I respectively, so $R_{\mathrm{H}^{+}} \sim 210 R_{\odot}$ and $R_{\mathrm{He}^{+}} \sim 205 R_{\odot}$. The $\mathrm{H} \beta$ flux from the eclipsed region, $F(\mathrm{H} \beta)=6.4 \times 10^{-13} \mathrm{ergs} \mathrm{cm}^{-2} \mathrm{~s}^{-1}$, suggests $n_{\mathrm{e}}=3 \times 10^{9} \mathrm{~cm}^{-3}$ for $T_{\mathrm{e}}=30000 \mathrm{~K}$.

The forbidden lines [O III] $\lambda \lambda 5007,4959$ and 4363 do not show intensity variations at primary minimum, although important variations along the eruption were observed. When it was possible to measure the three lines in our spectra, the reddening corrected line ratio $R=I(\lambda 5007)+I(\lambda 4959) / I(\lambda 4363)$ was calculated. In general, $R$ increases during the outburst, and extreme values such as 0.7 and 15.7 were measured at MJD 49945 and MJD 51065/70, respectively. The former value of $R$ corresponds to minimum values of $T_{\mathrm{e}, \min } \sim 16000 \mathrm{~K}$ and $n_{\mathrm{e}, \min } \sim 10^{7} \mathrm{~cm}^{-3}$ whereas the latter is consistent with $6000 \mathrm{~K} \leq T_{\mathrm{e}} \leq 40000 \mathrm{~K}$ and any values of $n_{\mathrm{e}}$. Generally, an increase in $R$ implies a decreases in $n_{\mathrm{e}}$ if $T_{\mathrm{e}}$ is held constant, and decreases in $T_{\mathrm{e}}$ if $n_{\mathrm{e}}$ is held constant. The [O III] emission line flux significantly increases during the outburst with a local minimum around MJD $51065 / 70$, coincinding with a drop in the He II 4686 emission line flux. A useful diagnostic for the changing physical conditions within the symbiotic nebula is provided by the $\mathrm{He} \mathrm{I} \lambda \lambda$ 5876, 6678, 7065 emission line ratios (Proga et al. 1994). In FN Sgr, the He I ratios change significantly during the outburst, in particular, both $I(\lambda 6678) / I(\lambda 5876)$ and $I(\lambda 7065) / I(\lambda 5876)$ decrease during the optical maximum. Similar outburst evolution of the He I lines has been noted in a few other S-type systems, and all of them show - like FN Sgr - relatively cool A or F-type optical continua throughout the outburst. This evolution of He I lines can be best explained by a decrease in the average $n_{\mathrm{e}}$ of the nebula (Proga et al. 1994). Such a change seems plausible if the hot component produces more He-ionizing photons and ionizes a larger region of lower average density.
Observations of CI Cyg and AX Per suggest that the $\mathrm{He}^{+}$ region actually shrinks in the orbital plane during their optical eruptions (Mikołajewska 1985; Mikołajewska \& Kenyon 1992), probably due to the presence of an optically and geometrically thick accretion disc which prevents ionization and excitation in the orbital plane. Unfortunately, our spectroscopic data are unsufficient to make a similar analysis for FN Sgr, however the similarity of the outburst evolution of the visual light curves of FN Sgr and those of CI Cyg suggests that the same may happen in FN Sgr. The apparent decrease in nebular size in the orbital plane then implies a large increase in nebular size perpendicular to the orbital plane, accompanied by a decrease in the average nebular density. Such a scenario is consistent with the observed evolution of the [O III] and He I emission lines.

If we assume that the [O III] lines arise from a homogeneous nebula with $T_{\mathrm{e}}=6000-16000 \mathrm{~K}$ and $n_{\mathrm{e}}=3 \times 10^{8}-10^{7} \mathrm{~cm}^{-3}$, the size of the $[\mathrm{O} \mathrm{III]} \mathrm{emitting} \mathrm{region}$ can be calculated (Mikołajewska 1985) using the unreddened flux of the $\lambda 5007$ line and adopting the distance $d=7.5 \mathrm{kpc}$. We obtain a range for the radius of 3-16 AU which is larger than the whole binary size and this may well explain the lack of eclipses in the [O III] lines. As in CI Cyg and AX Per, the [Fe VII] forbidden lines are the other emission lines which are unaffected by eclipses.

Two broad emission lines, $\lambda 6825$ and $\lambda 7082 \AA$, are frequently observed in symbiotic stars with high excitation nebulae and they are formed by Raman scattering of the OVI $\lambda \lambda 1031,1038$ resonance lines by neutral hydrogen (Schmid 1989). Only a weak and broad emission at $\lambda 6825 \AA$ is observed in several spectra of FN Sgr. The other feature is not detectable in the noisy region at the end of the aperture. No changes with the orbital phase are detected but during the period MJD 51325-51791, at the maximum of the light curve, the line $\lambda 6825 \AA$ is absent and the intensity increases when the brightness decreases, recovering almost the same intensity observed before the eruption. A probable explanation is alterations suffered by the scattering mechanism in the circumstellar medium during the explosion. Raman scattering is probably not working or it is less efficient because the UV photons are quickly absorbed by thick and expanded material around the hot component.

\section{Hot component}

The effective temperature and the luminosity of the hot component can be obtained from certain unreddened emission line fluxes. We can determine the hot component temperature using the relationship between $T_{\mathrm{h}}$ and the fluxes of $\mathrm{H} \beta$, He I 4471 and He II 4686 (Iijima 1981) for data acquired outside eclipse (Table 5). The second column of Table 5 includes $\chi_{\max }$, the highest observed ionization potential which is $\chi_{\max } \sim T_{\mathrm{h}}[1000 \mathrm{~K}]$ according to Mürset \& Nussbaumer (1994). To derive the hot component luminosity the following methods are used.

1. $L_{\mathrm{h}}(\mathrm{H} \beta)$ and $L_{\mathrm{h}}(\mathrm{He}$ II) are derived from $\mathrm{H} \beta$ and He II 4686 emission line fluxes adopting $T_{\mathrm{h}}$ for the 

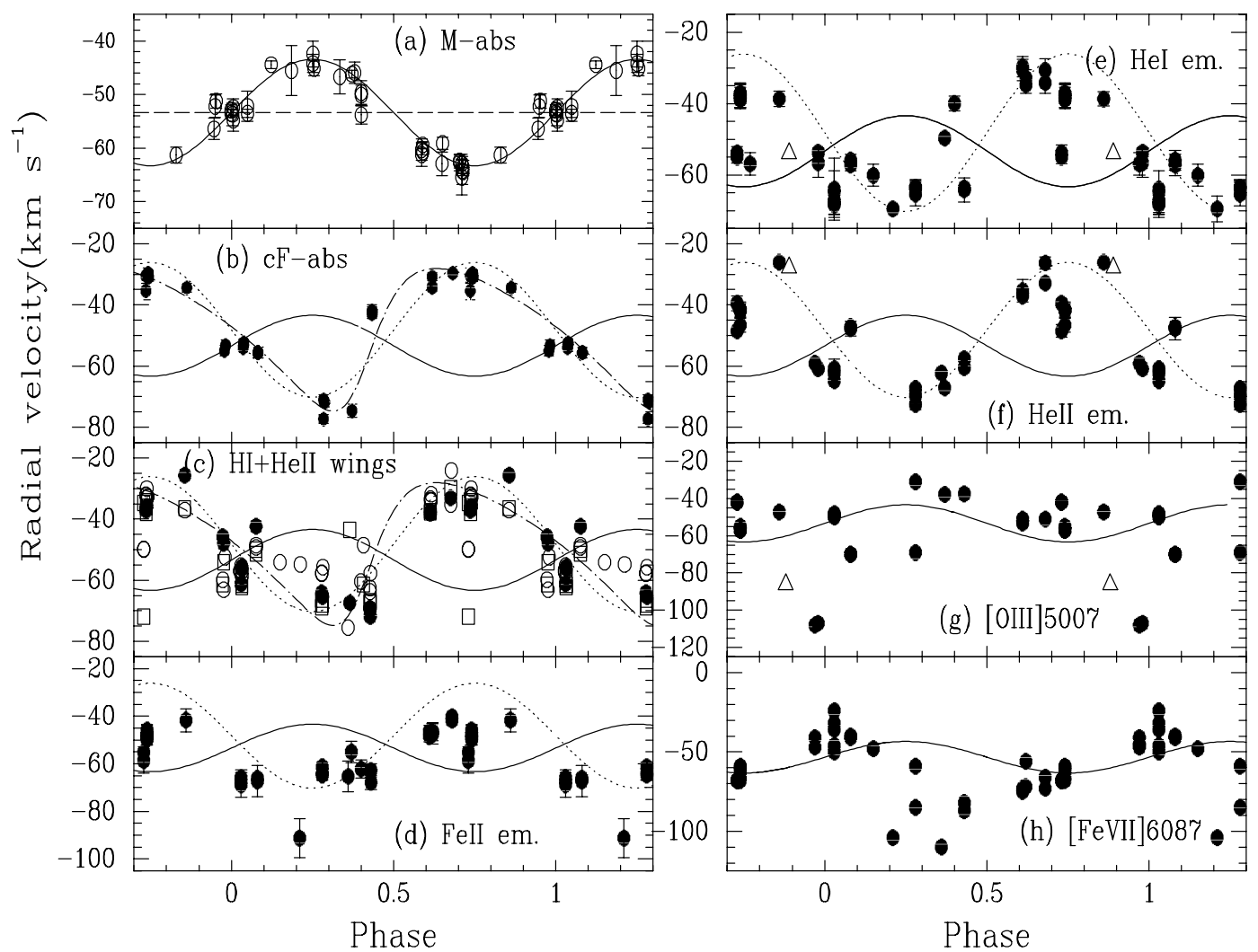

Fig. 6. Radial velocity data and orbital solutions for FN Sgr. The solid and dotted lines repeat the circular orbit of the M-giant and the cF-abs features, respectively. The dashed lines gives the best elliptical fit to the blue absorption system $\left(e=0.33 \pm 0.05, \omega=240^{\circ} \pm 9\right)$. Triangles represent Van Winckel's data. The solutions correspond to a) M giant absorptions, b) cF-absorption lines, c) H $\alpha$ (open circles), H $\beta$ (open squares) and He II $\lambda 4686$ wings (solid circles), d) Fe II emission lines, e) He I emission lines, f) He II emission lines, g) [O III] $\lambda 5007$ line and h) $[\mathrm{Fe}$ VII $] \lambda 6085$ line.

Table 4. Orbital solutions for FN Sgr.

\begin{tabular}{lrccccccc}
\hline \hline Component & $\begin{array}{c}K \\
{\left[\mathrm{~km} \mathrm{~s}^{-1}\right]}\end{array}$ & $\begin{array}{c}\gamma_{0} \\
{\left[\mathrm{~km} \mathrm{~s}^{-1}\right]}\end{array}$ & $e$ & $\begin{array}{c}\omega \\
{[\mathrm{deg}]}\end{array}$ & $\begin{array}{c}T_{0}^{1} \\
{[\mathrm{JD} 24 \ldots]}\end{array}$ & $\begin{array}{c}\Delta T^{2} \\
{[\text { day] }}\end{array}$ & $\begin{array}{c}a \sin i \\
{[\mathrm{AU}]}\end{array}$ & $\begin{array}{c}f(M) \\
{\left[M_{\odot}\right]}\end{array}$ \\
\hline M giant & $10.0 \pm 0.3$ & $-53.4 \pm 0.2$ & $0^{3}$ & & & 2.2 & 0.52 & 0.0580 \\
M giant & $10.0 \pm 0.3$ & $-53.4 \pm 0.2$ & $0.06 \pm 0.03$ & $368 \pm 29$ & 50995 & 7.1 & 0.52 & 0.0586 \\
cF-abs & $22.0 \pm 1.5$ & $-48.2 \pm 0.9$ & $0^{3}$ & & 50718 & -24.8 & 1.15 & 0.6300 \\
cF-abs & $23.3 \pm 1.0$ & $-47.5 \pm 0.6$ & $0.33 \pm 0.05$ & $240 \pm 9$ & 51614 & -16.4 & 1.15 & 0.6259 \\
He II wings & $16.8 \pm 2.0$ & $-51.3 \pm 1.2$ & $0^{3}$ & & & 29.0 & 0.88 & 0.2795 \\
He II wings & $18.3 \pm 1.4$ & $-51.6 \pm 1.1$ & $0.33 \pm 0.10$ & $271 \pm 15$ & 53424 & 26.8 & 0.90 & 0.3042 \\
H $\alpha+\mathrm{H} \beta$ wings & $11.0 \pm 1.3$ & $-51.2 \pm 0.8$ & $0^{3}$ & & & -19.1 & 0.57 & 0.0783 \\
Fe II & $9.3 \pm 1.8$ & $-60.0 \pm 1.8$ & $0^{3}$ & & & -75.3 & 0.48 & 0.0468 \\
He I & $15.8 \pm 1.0$ & $-54.1 \pm 0.8$ & $0^{3}$ & & & -32.2 & 0.82 & 0.2310 \\
He II & $16.5 \pm 1.3$ & $-53.8 \pm 0.8$ & $0^{3}$ & & & -33.1 & 0.86 & 0.2656 \\
[Fe VII] $\lambda 6087$ & $21.7 \pm 2.9$ & $-66.2 \pm 1.8$ & $0^{3}$ & & & 95.2 & 1.13 & 0.6028 \\
\hline
\end{tabular}

1 Time of the passage through periastron.

${ }^{2} \Delta T=T_{\text {sp conj }}-T_{\text {phot min }}$.

3 Assumed.

temperature and using Eqs. (6) and (7) given by Mikołajewska et al. (1997).

2. $L_{\mathrm{h}}(\mathrm{UV})$ is estimated from $\mathrm{H} \beta$, HeI5876, and He II 4686 emission line fluxes using Eq. (8) in Kenyon et al. (1991).

3. $L_{\mathrm{h}}(\mathrm{A}-\mathrm{F})$ is derived from the visual magnitude of the eclipsed A-F-type continuum. Note that $L_{\mathrm{h}}(\mathrm{A}-\mathrm{F})$, derived for the period of visual maximum (JD $2450590-51000$ ), is consistent with the results of the continuum fitting (bf+ff nebular emission plus black body continuum with $T_{\text {eff }}=$ $8000 \mathrm{~K}$ and $L=1000 L_{\odot}$ ) for the low resolution spectrum made on JD 2450590.

Figure 7 shows the evolution of the hot component in the HR diagram. We separated the observations in four intervals 
Table 5. Hot component of FN Sgr.

\begin{tabular}{lcccccc}
\hline \hline $\mathrm{JD}^{1} /$ phase & \multicolumn{3}{c}{ Temperature } & \multicolumn{5}{c}{ Luminosity $^{2}\left[L_{\odot}\right]$} \\
& $\chi_{\max }[\mathrm{eV}]$ & $T_{\text {el }}\left[10^{3} \mathrm{~K}\right]$ & $L_{\mathrm{h}}(\mathrm{H} \beta)$ & $L_{\mathrm{h}}(\mathrm{He}$ II 4686$)$ & $L_{\mathrm{h}}(\mathrm{UV})$ & $L_{\mathrm{h}}(\mathrm{A}-\mathrm{F})$ \\
\hline $48117 / 0.212^{3}$ & 114 & & & & & \\
$48190 / 0.360^{3}$ & 114 & 170 & 1460 & 1800 & 1440 & \\
$48203 / 0.366^{3}$ & 114 & 150 & 1080 & 1200 & 430 & \\
$49945 / 0.428$ & 114 & 150 & 1200 & 1500 & 1200 & 540 \\
$50590 / 0.563$ & 55 & 70 & 1530 & 1500 & 1080 & 1940 \\
$51065-70 / 0.402$ & 55 & $58:$ & 1420 & $945:$ & 1000 & 1940 \\
$51325 / 0.856$ & 55 & 127 & 1030 & 1240 & 1080 & 970 \\
$51755-7 / 0.614$ & 100 & 160 & 1420 & 1800 & 1440 & 1400 \\
$51791 / 0.686$ & 100 & 180 & 1900 & 2700 & 2200 & 1620 \\
$51826-8 / 0.738$ & 114 & 150 & 1850 & 2400 & 1800 & 540 \\
$52133-5 / 0.279$ & 114 & 170 & 1170 & 1550 & 1200 & 430 \\
\hline
\end{tabular}

${ }^{1}$ Julian Date $=2400000+\mathrm{JD}$ listed in table; ${ }^{2}$ assumes $d=7.5 \mathrm{kpc}$ and $E_{B-V}=0.2$;

${ }^{3}$ Based on emission line fluxes from Barbá et al. (1992).

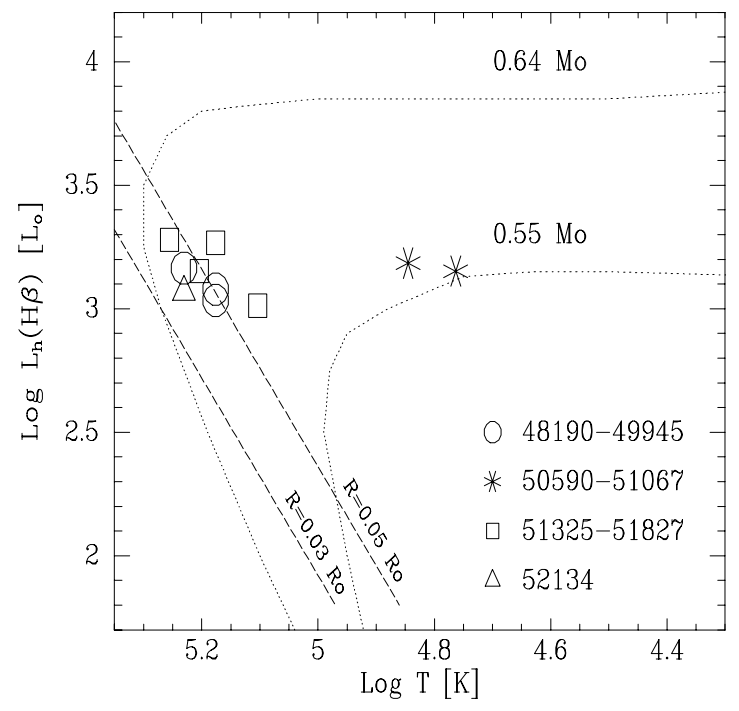

Fig. 7. Evolution of the hot component in the HR diagram. The observations are separated into four intervals covering the outburst and its preceeding and subsequent quiescent periods as indicated in the legend. The dotted curves are the cooling curves for a $0.64 M_{\odot}$ and $0.55 M_{\odot}$ white dwarf (Schönberner 1989) and the dashed curves are the line of constant radius.

as indicated in the legend. We do not observe any important variation in the luminosity whereas its temperature varies from $\log T_{\mathrm{h}} \sim 5.2$ to 4.7 being the lowest temperature at the maximum outburst. This behavior does not correspond with thermonuclear models and a possible explanation arguments in favour of accretion disk instabilities (Mikołajewska 2003), similar to those of Z And (Mikołajewska \& Kenyon 1996).

If the hot component is a low mass star surrounded by an accretion disk, similar considerations to those performed for CI Cyg (Kenyon et al. 1991) and AX Per (Mikołajewska \& Kenyon 1992) can be made. The disk models predict that about one-half of the accretion energy is radiated by the accretion disk itself, but the rest must be radiated in a boundary layer between the disk and the central star. Since the temperature in this region $\left(T_{\mathrm{bl}}\right)$ can be higher than $10^{5} \mathrm{~K}$, it emits mostly in the EUV range. If the radiation emitted shortward of $1200 \AA$ is produced by photoionization followed by recombination, the luminosity $L_{\mathrm{h}}(\mathrm{UV})$ can be approximated as the sum of the $\mathrm{HI}$, $\mathrm{He} \mathrm{I}$ and He II Lyman continua inferred from $\mathrm{H} \beta$, He I 5876, and He II 4686 emission line fluxes acording to Eq. (8) of Kenyon et al. (1991).

The radius of the accreting star and the mass accretion rate $\dot{M}$ can be calculated in base of Eqs. (9) and (10) of Kenyon et al. (1991). If $T_{\mathrm{bl}}=T_{\mathrm{h}}, L_{\mathrm{bl}}=0.5 L_{\mathrm{h}}$ and $M_{\mathrm{h}}=0.7 M_{\odot}$ we obtain $R_{\mathrm{h}} \sim 0.2 R_{\odot}$ which corresponds to a white dwarf with $0.7 M_{\odot}$. During the maximum brigthness (JD 50 590) $R_{\mathrm{h}} \sim 0.7-0.9 R_{\odot}$ and $\dot{M}_{\mathrm{h}} \sim 4 \times 10^{-5} M_{\odot} \mathrm{yr}^{-1}$.

\section{Conclusions}

FN Sgr is an eclipsing symbiotic binary with a period of 568.3 days determined photometrically and confirmed by spectroscopic observations. The present study of FN Sgr may be summarized as follows:

(i) The collected historical 1972-2002 light curve of FN Sgr shows systematic brightness decline with periodic deep minima (eclipses), interrupted by one major eruption around JD 2451000.

(ii) We have determined for the first time spectroscopic orbits based on the radial velocity curves for the cool and the hot stars. The radial velocity of both the cF-type absorption lines due to singly ionized elements and the He II broad emission wings seem to follow the hot component motion. Based on the light curve, we derive the red giant's radius $\left(140 R_{\odot}\right)$ and the orbital inclination $\left(i=80^{\circ}\right)$. We find that FN Sgr is similar to other S-type symbiotic binaries, composed of an M 5-type giant $\left(M_{\mathrm{g}}=1.5 M_{\odot}\right)$ and a hot white dwarf $\left(M_{\mathrm{h}}=0.7 M_{\odot}, R_{\mathrm{h}}=0.2 R_{\odot}\right)$ with a binary separation of $\sim 1.6 \mathrm{AU}$. The process of mass transfer in this system is probably through Roche lobe overflow since we find that the Roche lobe filling factor, considered as the ratio of the red giant's radius to the mean tidal radius $\left(R_{\mathrm{t}}\right)$, is near unity. 
(iii) The best solution for the cF-type absorption features (as well as that for the He II broad emission line wings) leads to significant eccentricity ( $e=0.33$ ) whereas the red giant orbit is circular or nearly circular. We note that a similar result was obtained for AR Pav, in which the eccentricity apparently increases with the hot component activity. Moreover, in both FN Sgr and AR Pav the periastron longitude of the elliptical orbit solution for the hot component is near $270^{\circ}$ indicating that the apparent eccentricity may be an effect tied to the line of sight.

(iv) The emission line changes indicate a decrease in the hot component temperature at the optical maximum to $\leq 50000 \mathrm{~K}$, possibly because of increasing optical depth in the hot component wind. The optical continuum observed during the 1996-98 maximum is consistent with a presence of continuum source with $T_{\text {eff }} \sim 7000-8000 \mathrm{~K}$ and $L \sim 2000-1000 L_{\odot}$.

(v) The presence of the blue shell absorption system apparently associated with the hot component in all our spectra, including periods where the strong high-ionization features are present, suggests a double-temperature structure of the hot component that is observed in other classical symbiotic stars. They possibly originated in a geometrically and optically thick accretion disk around a low-mass accretor. The evolution of $T_{\mathrm{h}}$ and $L_{\mathrm{h}}$ along the active phase argues in favour of accretion disk instabilities similar to those of Z And.

Acknowledgements. Part of this work was supported by the German Deutsche Forschungsgemeinschaft, DFG project number Ts $17 / 2-1$, and by the Polish KBN Research Grant No. 1P03D 017 27. The CCD and data acquisition system at CASLEO has been partly finance by R. M. Rich through US NSF grant AST-90-15827.

\section{References}

Barbá, R., Brandi, E., García, L., \& Ferrer, O. 1992, PASP, 104, 330 Belczyński, K., Mikołajewska, J., Munari, U., Ivison, R. J., \& Friedjung, M. 2000, A\&AS, 146, 407

Bertiau, F. C. 1967, IAU Symp., 30, 227
Burstein, D., \& Heiles, C. 1982, AJ, 87, 1165

Cahn, J. M. 1980, Space Sci. Rev., 457

Eggleton, P. P. 1983, ApJ, 268, 368

Hamuy, M., Walker, A. R., Suntzeff, N. B., et al. 1992, PASP, 104, 533

Hamuy, M., Suntzeff, N. B., Heathcote, S. R., et al. 1994, PASP, 106, 566

Hollis, J. M., Pedelty, J. A., \& Lyon, R. G. 1997, ApJ, 482, L85

Iijima, T. 1981, in Photometric and spectroscopic binary systems, ed. E. B. Carling, \& Z. Kopal (Dordrecht: Reidel)

Jung, Y.-C., \& Lee, H.-W. 2004, MNRAS, 350, 580

Kenyon, S. J., \& Fernandez-Castro, T. 1987, AJ, 93, 938

Kenyon, S. J., Oliversen, N. A., Mikołajewska, J., et al. 1991, AJ, 101, 637

Mikołajewska, J. 1985, Acta Astron., 35(1-2)

Mikołajewska, J., \& Kenyon, S. J. 1992, AJ, 103, 579

Mikołajewska, J., \& Kenyon, S. J. 1996, AJ, 112, 1659

Mikołajewska, J. 1997a, Physical Processes in Symbiotic Binaries and Related Systems, Copernicus Foundation for Polish Astronomy, Warsaw, 3

Mikołajewska, J., Acker, A., \& Stenholm, B. 1997, A\&A, 327, 191

Mikołajewska, J. 2001, in Small-Telescope Astronomy on Global Scales, ed. B. Paczyński, W. P. Chen, \& C. Lemme, ASP Conf. Ser., 246, 167

Mikołajewska, J. 2003, in Symbiotic stars probing stellar evolution, ed. R. L. M. Corradi, J. Mikołajewska, \& T. J. Mahoney, ASP Conf. Ser., 303, 9

Munari, U., Yudin, B. F., Taranova, O. G., et al. 1992, A\&AS, 93, 383

Munari, U., \& Buson, L. M. 1994, A\&A, 287, 87

Mürset, U., \& Nussbaumer, H. 1994, A\&A, 282, 586

Payne, C. H. 1928, Bull. Harv. Coll. Obs., 861, 8

Proga, D., Mikołajewska, J., \& Kenyon, S. J. 1994, MNRAS, 268, 213

Quiroga, C., Mikołajewska, J., Brandi, E., Ferrer O. E., \& García, L. G. 2002, A\&A, 387, 139

Schild, H., \& Schmid, H. M. 1996, A\&A, 310, 211

Schild, H., Dumm, T., Mürset, U., et al. 2001, A\&A, 366, 972

Schmid, H. M. 1989, A\&A, 221, L31

Schneider, D. P., \& Young, P. 1980, ApJ, 238, 946

Schönberner, D. 1989, IAU Symp., 131, 463

Schwarzenberg-Czerny, A. 1997, ApJ, 489, 941

Shafter, A. W. 1983, ApJ, 267, 222

Van Winckel, H., Duerbeck, H. W., \& Schwarz, H. E. 1993, A\&AS, 102, 401 
E. Brandi et al.: Spectroscopic orbits of the symbiotic binary FN Sgr, Online Material p 1

\section{Online Material}


Table 1. Log of the spectroscopic observations of FN Sgr.

\begin{tabular}{lccc}
\hline \hline Date & $\begin{array}{c}\text { JD } \\
2440000+\end{array}$ & $\begin{array}{c}\text { Range } \\
{[\AA]}\end{array}$ & $\begin{array}{c}\text { Phase } \\
(*)\end{array}$ \\
\hline $14 / 08 / 90(\dagger)$ & 8117.51 & $5800-7200$ & 0.212 \\
$06 / 11 / 90(\dagger)$ & 8201.50 & $5800-7200$ & 0.360 \\
$09 / 11 / 90(\dagger)$ & 8204.50 & $4400-5000$ & 0.365 \\
$14 / 08 / 95^{1}$ & 9944.60 & $4150-7300$ & 0.427 \\
$14 / 08 / 95^{1}$ & 9944.64 & $4150-7300$ & 0.427 \\
$14 / 08 / 95^{1}$ & 9944.66 & $4150-7300$ & 0.427 \\
$22 / 05 / 97(\dagger)$ & 10590 & $3540-7425$ & 0.563 \\
$22 / 05 / 97(\dagger)$ & 10590 & $3099-5098$ & 0.563 \\
$07 / 09 / 98^{1}$ & 11064.65 & $5700-9000$ & 0.398 \\
$12 / 09 / 98^{2}$ & 11069.63 & $4100-7100$ & 0.407 \\
$25 / 05 / 99^{1}$ & 11324.83 & $4500-7600$ & 0.856 \\
$31 / 08 / 99^{1}$ & 11421.64 & $5500-9100$ & 0.026 \\
$31 / 08 / 99^{1}$ & 11421.66 & $5500-9100$ & 0.026 \\
$02 / 09 / 99^{1}$ & 11423.59 & $4100-7300$ & 0.030 \\
$03 / 09 / 99^{1}$ & 11424.65 & $4100-7300$ & 0.032 \\
$03 / 09 / 99^{1}$ & 11424.67 & $4100-7300$ & 0.032 \\
$28 / 07 / 00^{1}$ & 11754.74 & $3950-7100$ & 0.613 \\
$28 / 07 / 00^{1}$ & 11754.76 & $3950-7100$ & 0.613 \\
$30 / 07 / 00^{1}$ & 11756.74 & $5500-8700$ & 0.616 \\
$30 / 07 / 00^{1}$ & 11756.76 & $5500-8700$ & 0.616 \\
$02 / 09 / 00^{1}$ & 11790.66 & $3900-7100$ & 0.676 \\
$03 / 09 / 00^{1}$ & 11791.67 & $5500-8700$ & 0.678 \\
$07 / 10 / 00^{1}$ & 11825.57 & $4200-7300$ & 0.737 \\
$07 / 10 / 00^{1}$ & 11825.58 & $4200-7300$ & 0.737 \\
$08 / 10 / 00^{1}$ & 11826.58 & $4200-7300$ & 0.739 \\
$09 / 10 / 00^{1}$ & 11827.56 & $5800-9000$ & 0.741 \\
$09 / 10 / 00^{1}$ & 11827.57 & $5800-9000$ & 0.741 \\
$17 / 04 / 01^{1}$ & 12017.88 & $3850-7100$ & 0.076 \\
$17 / 04 / 01^{1}$ & 12017.91 & $3850-7100$ & 0.076 \\
$29 / 05 / 01^{1}$ & 12059.88 & $5500-8600$ & 0.150 \\
$10 / 08 / 01^{1}$ & 12132.67 & $4100-7200$ & 0.278 \\
$10 / 08 / 01^{1}$ & 12132.68 & $4100-7200$ & 0.278 \\
$10 / 08 / 01^{1}$ & 12132.68 & $4100-7200$ & 0.278 \\
$12 / 08 / 01^{1}$ & 12134.66 & $4100-7200$ & 0.281 \\
$24 / 04 / 02^{1}$ & 12389.86 & $4800-7500$ & 0.730 \\
$24 / 04 / 02^{1}$ & 12389.91 & $4800-7500$ & 0.730 \\
$09 / 09 / 02^{1}$ & 12527.58 & $4200-7300$ & 0.972 \\
$11 / 09 / 02^{1}$ & 12529.57 & $4200-7300$ & 0.976 \\
$13 / 09 / 02^{1}$ & 12531.60 & $5400-8400$ & 0.980 \\
\hline 1070 & & &
\end{tabular}

(*) Photometric ephemeris $\operatorname{Min}\left(m_{\text {vis }}\right)=2450270+568.3$ E.

$(\dagger)$ Observations obtained with Z-Machine at CASLEO (Barbá et al. 1992).

(†) Low resolution spectra obtained with the $1.52 \mathrm{~m}$ telescope of ESO, La Silla, Chile.

1 Images taken with bin factor $=2$.

2 Image taken with bin factor $=4$. 
E. Brandi et al.: Spectroscopic orbits of the symbiotic binary FN Sgr, Online Material $p 3$

Table 2. Optical emission line fluxes in units of $10^{-13} \mathrm{erg} \mathrm{s}^{-1} \mathrm{~cm}^{-2}$, [TiO $]_{1}$ molecular index and $V_{\mathrm{sp}}$ magnitudes.

\begin{tabular}{|c|c|c|c|c|c|c|c|c|c|c|c|c|c|}
\hline 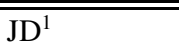 & -55 & 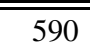 & $1065 / 70$ & 1325 & $1422 / 4$ & $1755 / 7$ & 1791 & $1826 / 8$ & 2018 & 2060 & $2133 / 5$ & 2390 & $2528 / 32$ \\
\hline$\phi$ & 0.428 & 0.563 & 0.402 & 0.856 & 0.029 & 0.614 & 0.677 & 0.738 & 0.075 & 0.150 & 0.279 & 0.730 & 0.976 \\
\hline $\mathrm{H} \gamma 4340$ & 9.5 & 18.5 & 14.3 & & 1.9 & 10.5 & 13.0 & 18.9 & 4.1 & & 7.2 & & 3.3 \\
\hline [O III] 4363 & 0.4 & 2.2 & 0.1 & & 1.8 & 3.8 & 4.7 & 3.7 & 2.3 & & 2.6 & & 1.1 \\
\hline He I 4388 & 1.0 & 2.1 & 1.2 & & 0.1 & 1.0 & 1.9 & 1.0 & 0.2 & & 0.6 & & \\
\hline He I 4471 & 1.0 & 4.5 & 2.6 & & $\ldots$ & 1.2 & 1.7 & 1.2 & 0.5 & & 0.4 & & 0.5 \\
\hline He II 4541 & 0.6 & $\ldots$ & $\ldots$ & & $\ldots$ & 0.6 & 1.5 & 0.9 & 0.5 & & 0.5 & & 0.4 \\
\hline Fe II 4584 & 0.4 & & $\ldots$ & $\ldots$ & 0.8 & 0.3 & 0.6 & 0.3 & $\ldots$ & & 0.2 & $\ldots$ & $\ldots$ \\
\hline Fe II 4629 & $\ldots$ & & 0.6 & 0.1 & 0.2 & 0.2 & 0.5 & 0.3 & $\ldots$ & & 0.1 & $\ldots$ & $\ldots$ \\
\hline N III 4634 & 0.3 & $\ldots$ & $\ldots$ & 0.2 & 0.7 & 1.6 & 0.8 & $\ldots$ & $\ldots$ & & $\ldots$ & $\ldots$ & $\ldots$ \\
\hline N III 4641 & 0.6 & $\ldots$ & $\ldots$ & $\ldots$ & $\ldots$ & 2.1 & 4.4 & 1.7 & 0.3 & & 0.4 & & 0.2 \\
\hline He II 4686 & 14.0 & 0.9 & 0.1 & 8.5 & 3.4 & 18.3 & 31.7 & 21.3 & 11.5 & & 17.1 & & 7.1 \\
\hline $\mathrm{H} \beta 4861$ & 25.1 & 42.7 & 41.4 & 25.0 & 5.5 & 29.7 & 36.5 & 39.2 & 9.4 & & 22.7 & 19.0 & 7.2 \\
\hline He I 4922 & 3.2 & 2.9 & 1.6 & 2.2 & 0.3 & 3.0 & 3.8 & 2.5 & 0.5 & & 1.7 & 1.0 & 0.2 \\
\hline Fe II 4924 & 0.3 & & 1.1 & 0.1 & $\ldots$ & 0.3 & 0.7 & 0.2 & 0.1 & & $\ldots$ & $\ldots$ & \\
\hline [O III] 4959 & $\ldots$ & & $\ldots$ & 3.2 & 1.4 & 2.4 & 2.2 & 1.1 & 0.6 & & 0.8 & 0.2 & 0.4 \\
\hline [O III] 5007 & 0.2 & 3.3 & 1.4 & 9.7 & 3.7 & 7.5 & 5.0 & 3.7 & 1.9 & & 2.3 & 0.9 & 1.0 \\
\hline He I 5016 & 1.7 & 3.6 & 2.8 & 2.2 & 0.4 & 1.6 & 2.0 & 1.2 & 0.5 & & 0.8 & 0.4 & 0.3 \\
\hline Fe II 5018 & 0.7 & & 1.1 & 0.2 & 0.2 & 0.6 & 1.1 & 0.6 & 0.1 & & 0.2 & 0.2 & ... \\
\hline He I 5047 & 0.6 & $\ldots$ & 0.8 & 9.6 & $\ldots$ & 0.3 & 1.4 & 1.1 & $\ldots$ & & $\ldots$ & 0.3 & $\ldots$ \\
\hline He II 5411 & 1.3 & $\ldots$ & $\ldots$ & 0.3 & 0.2 & 1.6 & 3.9 & 1.8 & 1.0 & & 1.4 & 1.3 & 0.7 \\
\hline$[\mathrm{Fe}$ VII $] 5720$ & 0.6 & $\ldots$ & $\ldots$ & $\ldots$ & $\ldots$ & 0.4 & 0.6 & 0.8 & 1.2 & 1.2 & 1.3 & 1.8 & 1.4 \\
\hline He I 5876 & 6.1 & 10.7 & 9.4 & 10.3 & 2.4 & 6.1 & 8.2 & 8.7 & 2.6 & 1.7 & 4.0 & 2.2 & 2.8 \\
\hline [Fe VII $] 6087$ & 0.9 & $\ldots$ & $\ldots$ & $\ldots$ & 0.3 & 0.7 & 1.1 & 1.1 & 2.0 & 2.3 & 2.8 & 2.9 & 2.2 \\
\hline Н $\alpha 6563$ & 127 & 207 & 180 & 192 & 43.0 & 118 & 208 & 187 & 51.0 & 58.7 & 105 & 84 & 44.9 \\
\hline $\mathrm{H} \alpha \mathrm{CA}^{3}$ & $\ldots$ & & 64 & 38 & 42 & 25 & 38 & 51 & 11 & 7.1 & 5.3 & $\ldots$ & 6.2 \\
\hline He I 6678 & 13.1 & 5.4 & 4.2 & 6.1 & 1.5 & 8.7 & 9.0 & 8.7 & 2.4 & 1.7 & 3.2 & 2.5 & 0.7 \\
\hline O VI 6825 & 2.7 & $\ldots$ & $\ldots$ & $\ldots$ & $\ldots$ & $\ldots$ & $\ldots$ & 2.5: & $\ldots$ & $0.5:$ & 2.3: & 2.4 & 1.1 \\
\hline He I 7065 & 6.6 & 5.5 & 4.9 & 10.2 & 2.3 & 6.6 & 8.5 & 10.1 & 3.8 & 1.9 & 3.9 & 2.2 & 2.7 \\
\hline He I 7281 & 2.5 & 1.3 & 0.8 & 1.8 & 0.3 & 1.5 & 2.3 & 1.6 & & 0.6 & 0.6 & 0.9 & 0.2 \\
\hline$[\mathrm{TiO}]_{1}$ & 0.27 & 0.075 & 0.21 & 0.30 & 0.71 & 0.45 & & 0.46 & 0.64 & 0.35 & 0.35 & 0.35 & 0.34 \\
\hline$V_{\mathrm{sp}}[\mathrm{mag}]$ & 12.5 & 11.4 & $11.3^{2}$ & 12.1 & 13.4 & 11.7 & 11.6 & 12.5 & 13.2 & 12.6 & 12.7 & 12.7 & 13.4 \\
\hline
\end{tabular}

${ }^{1}$ Julian Date $=2450000+$ JD listed in table; ${ }^{2}$ scaled to $V_{\mathrm{sp}}=m_{\mathrm{vis}} ;{ }^{3}$ central absorption. 


\section{E. Brandi et al.: Spectroscopic orbits of the symbiotic binary FN Sgr, Online Material $p 4$}

Table 3. Radial velocities (in $\mathrm{km} \mathrm{s}^{-1}$ ) of the giant absorption lines, the blue cF-type absorption lines, broad emission wings of $\mathrm{H}$ I and He II lines and permitted and forbidden emission lines in FN Sgr.

\begin{tabular}{|c|c|c|c|c|c|c|c|c|c|c|}
\hline JD/Phase & M-abs & cF-type & Broa & $\overline{\mathrm{d} \text { emi }}$ & ssion wings & $\overline{\text { Fe II }}$ & $\overline{\mathrm{He} \mathrm{I}}$ & $\overline{\text { He II }}$ & [OO III] & $\overline{[\mathrm{Fe} \mathrm{VII}]}$ \\
\hline $2440000+$ & & & $\mathrm{H} \beta$ & $\mathrm{H} \alpha$ & Не II $\lambda 4686$ & & & & $\lambda 5007$ & $\lambda 6087$ \\
\hline $7357.867^{\dagger} / 0.88$ & & & & -44 & & & & & & \\
\hline $7359.867^{\dagger} / 0.88$ & & & & & & & & & -86 & \\
\hline $7363.867^{\dagger} / 0.89$ & & & & & & & $-54 \pm 2(2)$ & & & \\
\hline $7364.867^{\dagger} / 0.89$ & & & & & -36 & & & $-28(1)$ & & \\
\hline $7779.867^{\dagger} / 0.62$ & & & & -61 & & & & & & \\
\hline $8117.505 / 0.21$ & & & & -56 & & $-84 \pm 9(2)$ & $-70(1)$ & & & -104 \\
\hline $8201.500 / 0.36$ & $-47 \pm 3(9)$ & & -43 & -81 & & $-65 \pm 6(12)$ & & $-62(1)$ & & -110 \\
\hline $8204.500 / 0.37$ & & $-75 \pm 2(71)$ & & & -68 & $-55 \pm 4(28)$ & $-50 \pm 3(6)$ & $-67(1)$ & -38 & \\
\hline $9944.597 / 0.43$ & $-50 \pm 2(12)$ & $-42 \pm 2(122)$ & -65 & -66 & -66 & & & & & - \\
\hline $9944.635 / 0.43$ & $-50 \pm 2(12)$ & $-42 \pm 2(143)$ & -64 & -64 & -65 & $-68 \pm 3(41)$ & $-64 \pm 2(10)$ & $-60 \pm 1(5)$ & & -82 \\
\hline $9944.662 / 0.43$ & $-54 \pm 2(15)$ & $-43 \pm 2(146)$ & -65 & -59 & -70 & $-63 \pm 3(22)$ & $-64 \pm 2(10)$ & $-58 \pm 3(5)$ & -37 & -87 \\
\hline $11064.648 / 0.40$ & $-46 \pm 1(38)$ & & & -58 & & $-62 \pm 4(7)$ & $-40 \pm 9(4)$ & & & -188 \\
\hline $11069.634 / 0.41$ & $-46 \pm 2(4)$ & & -60 & -47 & -63 & & & & & \\
\hline $11324.827 / 0.86$ & $-61 \pm 1(23)$ & $-34 \pm 1(59)$ & -32 & -35 & -33 & $-42 \pm 5(8)$ & $-39 \pm 2(9)$ & $-26(1)$ & -47 & \\
\hline $11421.636 / 0.03$ & $-54 \pm 1(72)$ & & & -55 & & $-70 \pm 8(3)$ & $-64 \pm 6(3)$ & & & -46 \\
\hline $11421.659 / 0.03$ & $-53 \pm 1(61)$ & & & -56 & & & $-67 \pm 4(4)$ & & & -24 \\
\hline $11423.592 / 0.03$ & $-54 \pm 2(10)$ & $-54 \pm 1(87)$ & & -51 & -54 & $-69 \pm 5(6)$ & $-65 \pm 3(9)$ & $-61 \pm 0.4(2)$ & -48 & -50 \\
\hline $11424.650 / 0.03$ & $-52 \pm 2(13)$ & $-52 \pm 1(93)$ & -52 & -55 & -52 & $-66 \pm 3(7)$ & $-68 \pm 3(10)$ & $-62 \pm 4(3)$ & -49 & -32 \\
\hline $11424.672 / 0.03$ & $-55 \pm 2(13)$ & $-53 \pm 1(83)$ & -56 & -56 & -51 & $-67 \pm 2(35)$ & $-68 \pm 3(10)$ & $-65(1)$ & -50 & -36 \\
\hline $11754.741 / 0.61$ & $-60 \pm 2(11)$ & $-34 \pm 1(68)$ & -25 & -33 & -36 & $-46 \pm 2(36)$ & $-30 \pm 2(10)$ & $-35 \pm 0.3(3)$ & -51 & -75 \\
\hline $11754.764 / 0.61$ & $-61 \pm 2(10)$ & $-31 \pm 1(69)$ & -24 & -32 & -37 & $-48 \pm 2(40)$ & $-31 \pm 2(8)$ & $-37 \pm 2(3)$ & -53 & -73 \\
\hline $11756.745 / 0.62$ & $-59 \pm 1(70)$ & & & -33 & & $-46 \pm 4(7)$ & $-33 \pm 3(3)$ & & & -72 \\
\hline $11756.763 / 0.62$ & $-61 \pm 1(63)$ & & & -30 & & $-47 \pm 4(8)$ & $-35 \pm 3(4)$ & & & -56 \\
\hline $11790.664 / 0.68$ & $-63 \pm 2(12)$ & $-30 \pm 1(51)$ & -27 & -38 & -34 & $-40 \pm 1(54)$ & $-31 \pm 2(9)$ & $-33 \pm 2(6)$ & -51 & -73 \\
\hline $11791.668 / 0.68$ & $-59 \pm 1(78)$ & & & -24 & & $-42 \pm 2(11)$ & $-34 \pm 3(4)$ & $-26 \pm 2(2)$ & & -66 \\
\hline $11825.572 / 0.74$ & & $-30 \pm 1(49)$ & -30 & -32 & -38 & $-46 \pm 2(24)$ & $-39 \pm 3(8)$ & $-42 \pm 1(4)$ & -57 & -66 \\
\hline $11825.585 / 0.74$ & & $-30 \pm 1(61)$ & -33 & & -37 & $-49 \pm 2(30)$ & $-37 \pm 3(11)$ & $-47 \pm 3(4)$ & -57 & -68 \\
\hline $11826.576 / 0.74$ & $-64 \pm 3(14)$ & $-31 \pm 1(59)$ & & -30 & -37 & $-50 \pm 2(34)$ & $-37 \pm 3(10)$ & $-42 \pm 2(4)$ & -55 & -62 \\
\hline $11827.559 / 0.74$ & $-64 \pm 1(68)$ & & & & & $-48 \pm 4(7)$ & $-38 \pm 2(3)$ & & & -59 \\
\hline $11827.572 / 0.74$ & $-64 \pm 1(57)$ & & & -34 & & $-48 \pm 2(8)$ & $-38 \pm 2(3)$ & $-42(1)$ & & -60 \\
\hline $12017.884 / 0.08$ & $-52 \pm 3(25)$ & $-56 \pm 2(129)$ & -35 & -48 & -40 & $-67 \pm 6(9)$ & $-56 \pm 3(8)$ & $-48 \pm 2(4)$ & -70 & -41 \\
\hline $12017.906 / 0.08$ & $-54 \pm 3(25)$ & $-56 \pm 2(138)$ & -34 & -49 & -41 & $-66 \pm 1(2)$ & $-57 \pm 3(7)$ & $-47 \pm 2(3)$ & -70 & -40 \\
\hline $12059.878 / 0.15$ & $-44 \pm 1(72)$ & & & -54 & & & $-60 \pm 2(4)$ & & & -48 \\
\hline $12132.668 / 0.28$ & $-42 \pm 2(15)$ & $-77 \pm 1(103)$ & -69 & -57 & -62 & $-64 \pm 2(5)$ & $-65 \pm 2(4)$ & $-70 \pm 0.4(2)$ & & \\
\hline 12 132.684/ 0.28 & $-44 \pm 2(13)$ & $-71 \pm 2(79)$ & -59 & -57 & -65 & $-61 \pm 2(5)$ & $-64 \pm 2(4)$ & $-73 \pm 1(3)$ & -69 & -85 \\
\hline $12134.660 / 0.28$ & $-45 \pm 1(50)$ & $-72 \pm 2(125)$ & -60 & -54 & -68 & $-65 \pm 2(13)$ & $-64 \pm 2(9)$ & $-67 \pm 2(4)$ & -31 & -59 \\
\hline $12389.860 / 0.73$ & $-63 \pm 2(80)$ & $-35 \pm 3(38)$ & & & & $-55 \pm 2(2)$ & $-54 \pm 3(6)$ & $-40(1)$ & -42 & -68 \\
\hline $12389.908 / 0.73$ & $-62 \pm 1(75)$ & $-31 \pm 2(33)$ & & & & $-59 \pm 5(2)$ & $-55 \pm 4(6)$ & $-48(1)$ & -42 & -68 \\
\hline $12527.583 / 0.97$ & $-56 \pm 2(33)$ & $-55 \pm 2(134)$ & & & & nd & $-57 \pm 1(7)$ & $-59 \pm 1(3)$ & -108 & -41 \\
\hline $12529.574 / 0.98$ & $-52 \pm 2(29)$ & $-53 \pm 2(154)$ & & & & nd & $-57 \pm 2(7)$ & $-61 \pm 1(3)$ & -107 & -47 \\
\hline $12531.601 / 0.98$ & $-51 \pm 1(119)$ & & & & & nd & $-54 \pm 4(3)$ & & & -45 \\
\hline
\end{tabular}

$\dagger$ Van Winckel's data (1993).

nd: non detected. 